\title{
Paléohydrologie du bassin sédimentaire du Sénégal Essai d'explication des nappes déprimées
}

\author{
Babacar Dieng Ministère de l'hydraulique, Dakar, Sénégal
}

Un modèle de simulation hydrologique est utilisé pour l'étude du comportement des nappes superficielles et sous-jacentes du bassin sédimentaire du Sénégal, sur une période allant de 18000 ans avant notre ère jusqu'à l'époque actuelle. Cette étude, dont le présent article est le résumé, a obtenu le $2^{e}$ prix Henri Milon de la Société hydrotechnique de France pour 1987 (ex-aequo avec l'étude de J.P. Bravard dont le résumé est publié dans le présent numéro).

L'hydrogéologie des nappes des formations sédimentaires de l'Afrique subsaharienne pose un certain nombre de problèmes spécifiques pour lesquels les applications jusqu'ici avancées ne reposent que sur des considérations théoriques; la réalité des phénomènes reste encore insuffisamment connue. Parmi ces problèmes nous pouvons citer ceux relatifs aux :

- nappes déprimées plus connues sous le nom de " nappe en creux " (J. Archambault 1960)

- processus d'alimentation des nappes par les eaux de pluie.

Pour ce qui est des nappes déprimées, elles constituent des anomalies piézométriques qui se caractérisent par:

- un état piézométrique de ces nappes apparamment stabilité

- une allure de la piézométrie en forme de cuvette avec des niveaux relativement bas dans la partie centrale, ce qui fait que malgré l'écoulement centripète des bordures de la nappe vers le centre, ce dernier reste encore déprimé.

De telles anomalies ont été essentiellement expliquées par :

- des séquelles des dernières variations du niveau marin sur la côte Atlantique de l'Afrique auxquelles se sont jointes des variations climatiques intervenues au cours de la même période; soit le Quaternaire récent $\approx 18000$ ans BP.

- un déséquilibre entre une alimentation négligeable ou hypothétique de ces nappes et une éventuelle reprise par évaporation plus significative qui aurait partiellement vidangé et provoqué la forme en creux de ces nappes.

Cette deuxième hypothèse d'explication des nappes en creux est justement un des arguments qui fait poser le problème de l'alimentation des nappes superficielles par les eaux de pluie. En effet il serait assez difficile de concilier :

- d'une part les notions de Réserve Utile (ou Facilement Utilisable) souvent utilisées dans les calculs de bilan hydrologique et de pluie efficace qui représentent le stock d'eau emmagasiné dans les tranches superficielles du sol et qui sert à compenser l'évapotranspiration en l'absence de pluie.

- et d'autre part une évapotranspiration qui soit en mesure de puiser jusqu'à la nappe qui par moment se situe à plus de $80 \mathrm{~m}$ en dessous du sol.

Le présent article résume quelques réflexions et résultats d'un travail de thèse qui à partir d'une étude quantitative sur les nappes du bassin sédimentaires du Sénégal essaie de préciser les hypothèses d'explications les plus plausibles à ces anomalies.

Paleohydrology of the sedimention basin in Sénégal - Essay relating to depressed nappes

An hydrological simulation model is used to study the bahaviour of superficial nappes of the sedimentation basin in Senegal, and covering a period of 18,000 years prior to our era. This study, summed up in this article, obtained the Henri Milon's second prize in 1987 from the Sociêté hydrotechnique de France (ex-eaquo with J.P. Bravard's study whose summary is published in this issue). 


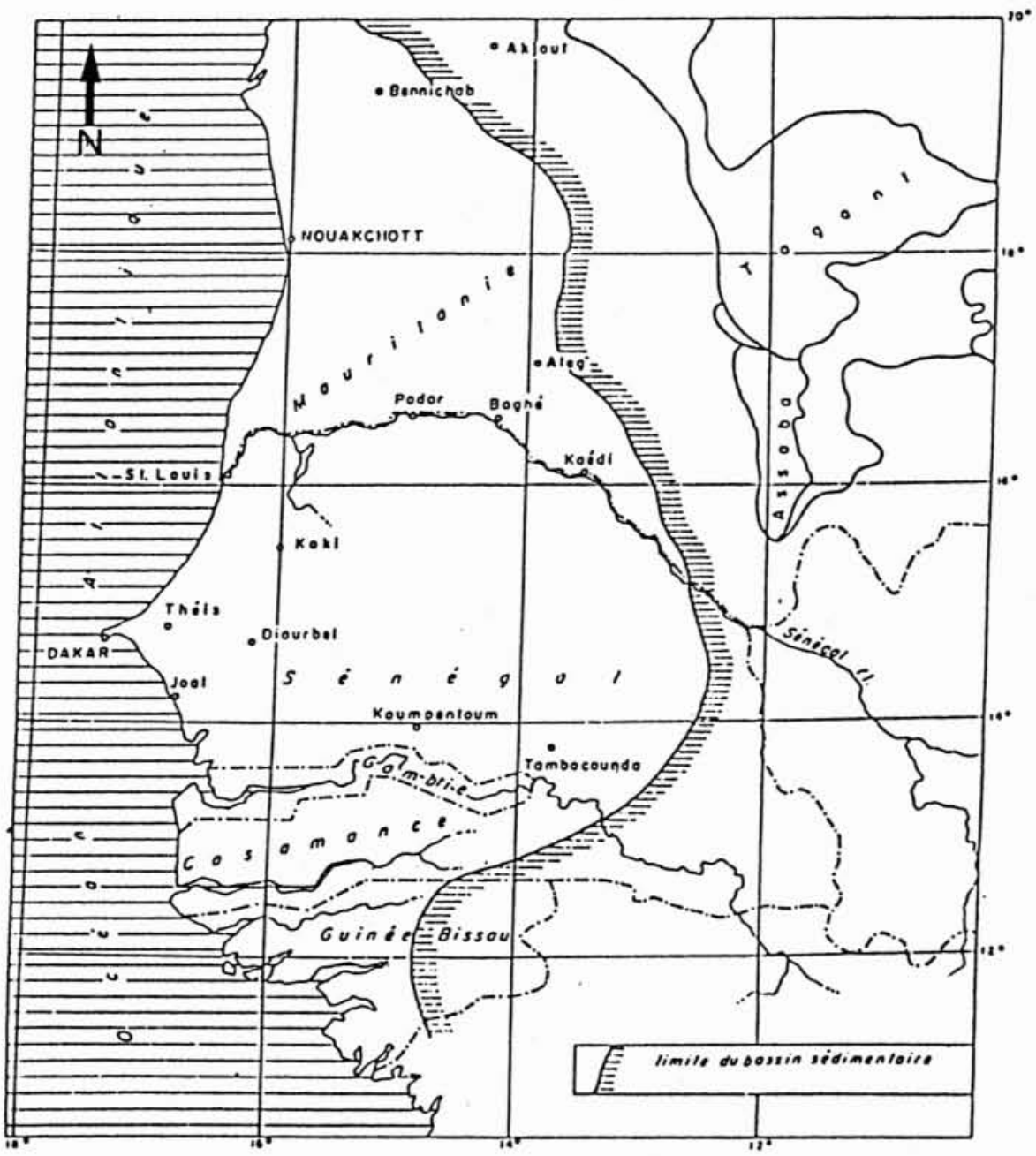

1. Localisation du bassin sédimentaire sénégalo-mauritanien.

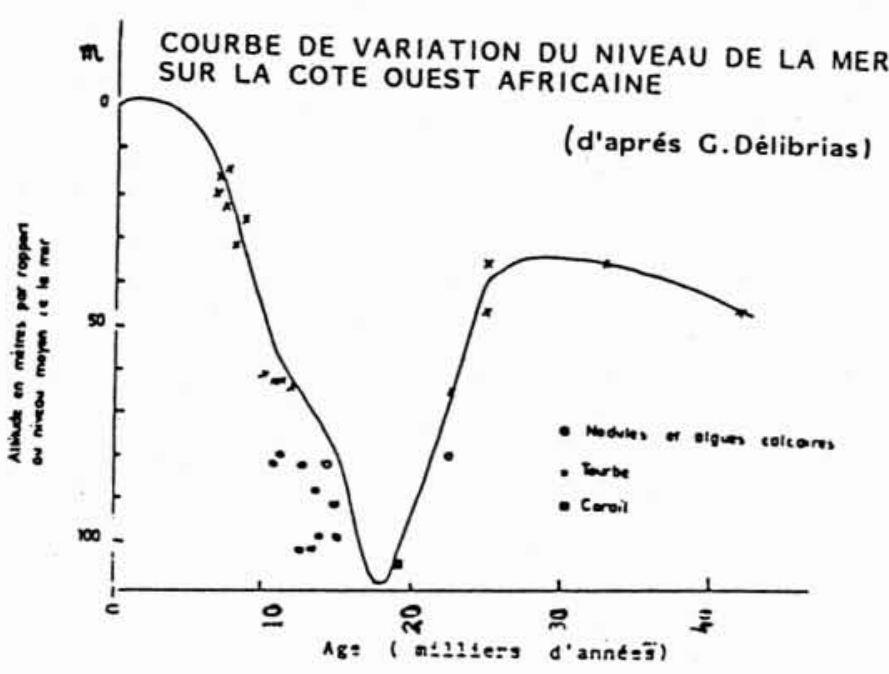

2. Variation du niveau marin pendant le dernier glaciaire de 40000 ans BP à l'actuel.

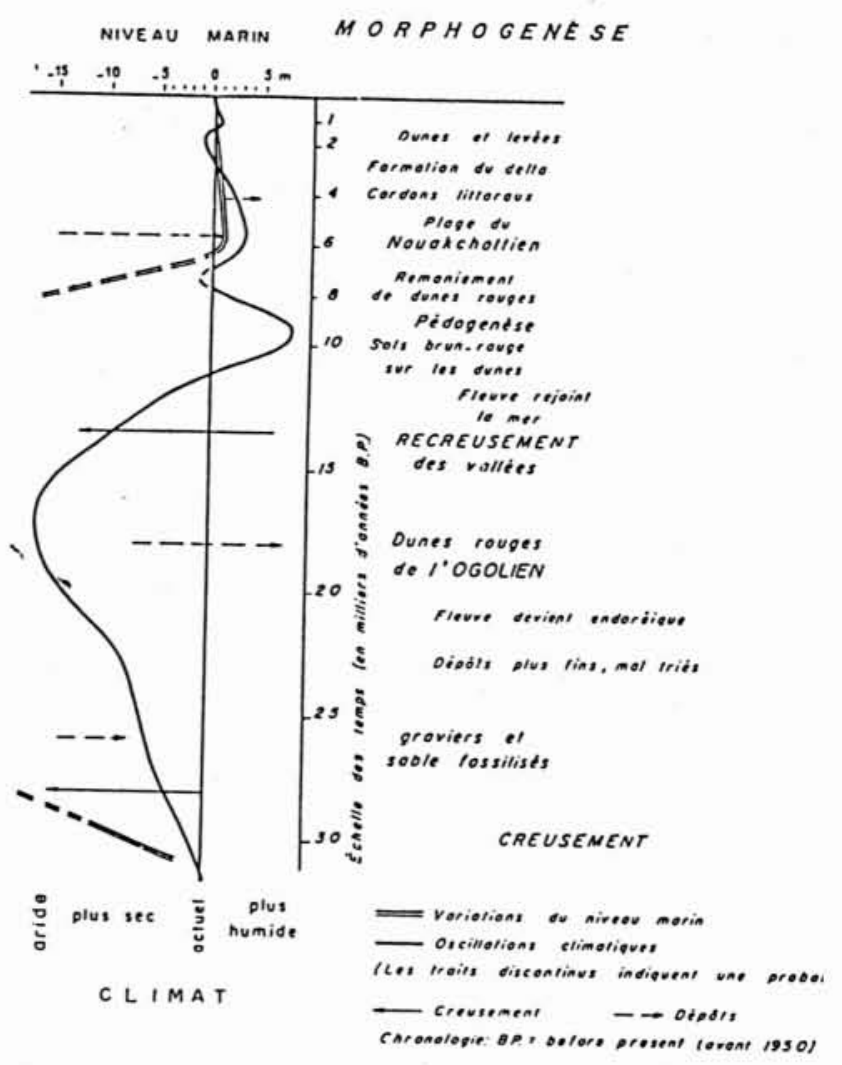

3. Évolution géomorphologique dans le bassin du fleuve Sénégal pendant le quaternaire récent (d'après $\mathrm{P}$. Michel 1973). 


\section{Domaine d'étude et travaux réalisés}

Il s'agit du bassin sédimentaire du Sénégal d'une superficie de près de $150000 \mathrm{~km}^{2}$ situé à la limite Ouest de l'Afrique Occidentale entre les $12^{\circ}$ et $16^{\circ}$ de Latitude Nord et qui fait partie d'un plus vaste ensemble dit bassin sédimentaire Sénégalo-Mauritanien (figure 1).

Les nappes superficielles de ce bassin contenues dans des formations tertiaires généralement sablo et grésoargileux, offrent dans leur partie centrale un exemple type de nappe en creux (figure 4). La pluviométrie moyenne annuelle répartie sur près de 4 mois qui passe de $1500 \mathrm{~mm}$ dans le Sud à moins de $400 \mathrm{~mm}$ dans le Nord, l'évapotranspiration relativement forte ( $>2000$ $\mathrm{mm}$ par an dans une bonne partie du bassin) et les faibles variations saisonnières du niveau des nappes, laissent entendre que les mécanismes d'infiltration des eaux de pluie revêtent une particularité liée à ces conditions climatiques.

Le travail réalisé a consisté à :

- tester de manière quantitative à l'aide d'un modèle de simulation hydrogéologique à structure multicouche maillée la plausibilité de l'hypothèse des variations du niveau marin et du climat comme explication de la nappe en creux du Ferlo du Sénégal.

- adapter parallèlement la notion de Réserve Utile du sol à partir de résultats expérimentaux relatifs à la dynamique de l'eau dans le sol, ceci en vue d'une meilleure quantification de la recharge des nappes.

- évaluer, à partir de nos résultats de simulation et d'estimation de l'évaporation sur nappe profonde (basée sur des méthodes isotopiques), l'importance de l'évaporation dans l'explication du phénomène de nappe en creux.

Cette explication de la nappe déprimée du Ferlo nous a permis de procéder à un bref tour d'horizon des différents cas de nappe en creux du reste de l'Afrique de l'Ouest et du Centre afin de leur apporter une explication.

\section{L'hypothèse des variations du niveau marin et du climat}

\subsection{Formulation}

La dernière partie du Quaternaire (700000 ans pour fixer les idées) a été marquée par une succession de périodes glaciaires et interglaciaires correspondant à des cycles de régression et de transgression marine. A chaque cycle, la période de régression marine, de durée relativement longue, a été suivie d'une transgression de très courte durée. Ainsi, au cours du dernier cycle glaciaire qui aurait commencé il $\mathrm{y}$ a près de 130000 ans, le niveau marin a subi une régression qui a duré près de 100000 ans, passant d'une côte équivalente à celle de l'actuel à près de $100 \mathrm{~m}$ plus bas (figure 2). La transgression qui suivait était de durée nettement plus courte (10 000 ans environ); ainsi entre 18000 ans BP et 8000 ans $\mathrm{BP}$, le niveau marin est repassé de son niveau le plus bas à sa cote actuelle. A ces variations eustatiques se sont jointes des variations du climat (figure 3) qui ont été d'extension plus étendue car elles ont touché toute la zone soudano-sahélienne et même équatoriale de l'Afrique. Ainsi la période de bas niveau marin correspondait à un maximum d'aridité du climat qui était tel qu'à l'échelle du Sénégal, les domaines morphoclimatiques étaient décalés de 4 à $5^{\circ}$ vers les basses latitudes (P. Michel 1973). En d'autres termes cela reviendrait à dire que les conditions climatiques actuelles de SaintLouis sont représentatives de celles de Ziguinchor il y a 18000 ans. Cet aridité du climat avait fait de sorte que les cours d'eau qui sont une source importante d'alimentation des nappes étaient soit affaiblis (Gambie, Casamance, Sénégal en amont de Bakel) soit sans écoulement (Sine, Saloum, vallée du Ferlo, Sénégal en aval de Bakel). Ainsi l'hyphothèse des variations du niveau marin et du climat pourrait se résumer de la façon suivante :

- pendant la dernière période de régression marine, le niveau bas de la mer a pu influencer de manière significative celui des nappes. La longue durée de celle-ci a permis une extension sensible vers l'intérieur du bassin touchant ainsi la vallée du Ferlo. En plus le climat sec et même aride de cette période n'a pas pu rehausser ces niveaux bas;

- au cours de la transgression suivante, la courte durée de cette dernière a fait que les niveaux initialement bas n'ont pu remonter que dans les zones peu distantes de la côte;

- le climat devenu humide vers 10000 ans BP a pu aussi relever le niveau des nappes dans la partie Sud et Sud-Est du bassin à pluviométrie plus forte et en bordure du fleuve Sénégal qui recommençait à couler dans sa partie aval en rejoignant la mer;

- sont donc restées déprimées les zones éloignées de la côte atlantique et des cours d'eau principaux mais aussi celles à pluviométrie relativement faible, en l'occurence le Ferlo.

En effet, en assimilant les variations du niveau marin à des variations brusques de charge qui se produisent à la limite du système hydrogéologique et qui se propagent par la suite à l'intérieur du bassin, on aperçoit déjà la logique de cette hypothèse. La solution à une dimension d'un tel problème s'écrit :

$$
h(x, t)=h_{0} \operatorname{erfc}\left(x \sqrt{\frac{S}{4 T t}}\right)
$$

avec :

$T$ et $S$ : transmissitivité et coefficient d'emmagasinement de la nappe

$h(x, t)$ : variation du niveau de la nappe après un instant $t$ à un point situé à la distance $x$ de la zone de variation brusque du niveau marin d'amplitude $h_{0}$

erfc : fonction erreur complémentaire.

Ainsi pour une nappe peu diffuse telle que celle du Continental Terminal du Ferlo (contenue dans des horizons sablo et gréso-argileux) ayant une transmissivité de l'ordre de $10^{-4} \mathrm{~m}^{2} / \mathrm{S}$ et un coefficient d'emmagasinement de l'ordre du pour cent, nous avons : 

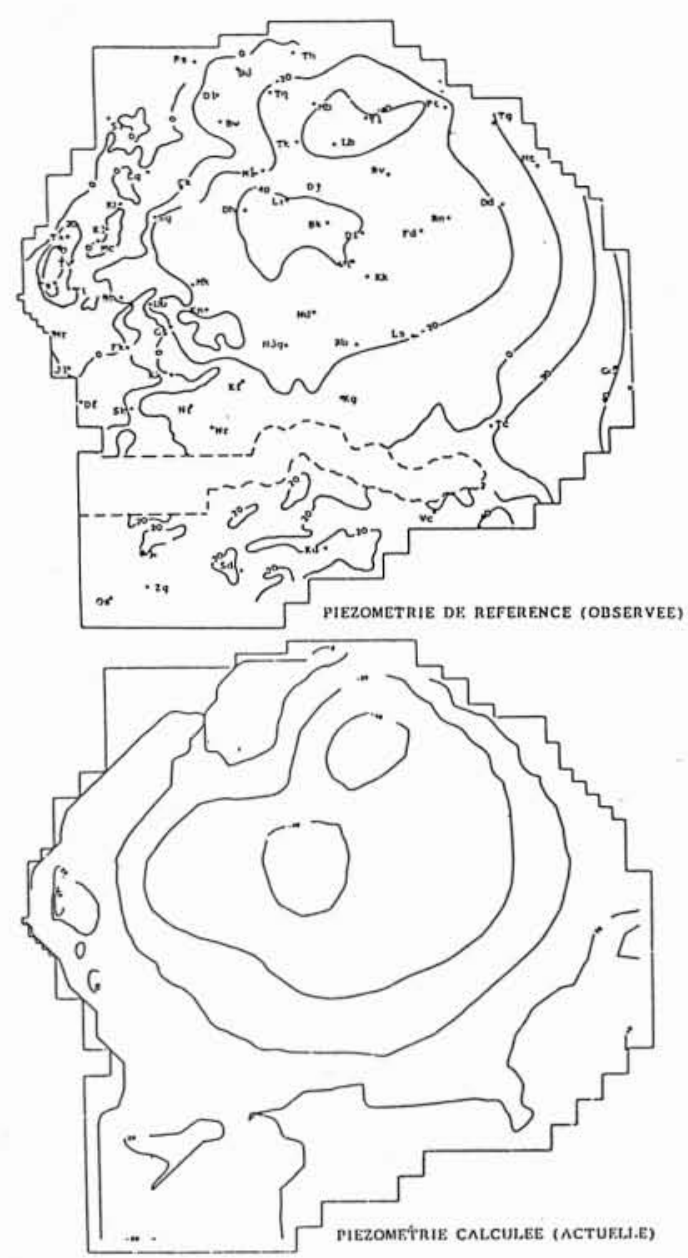

4. Nappes superficielles.
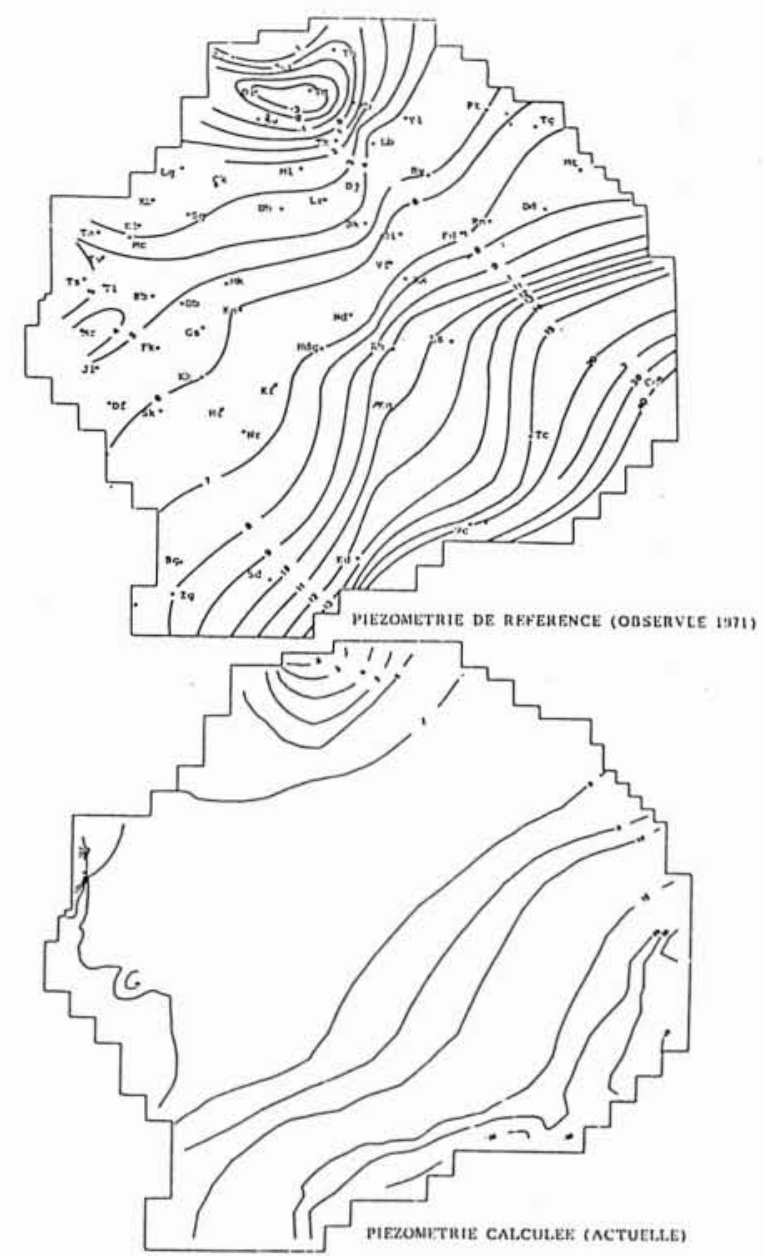

5. Nappe profonde.

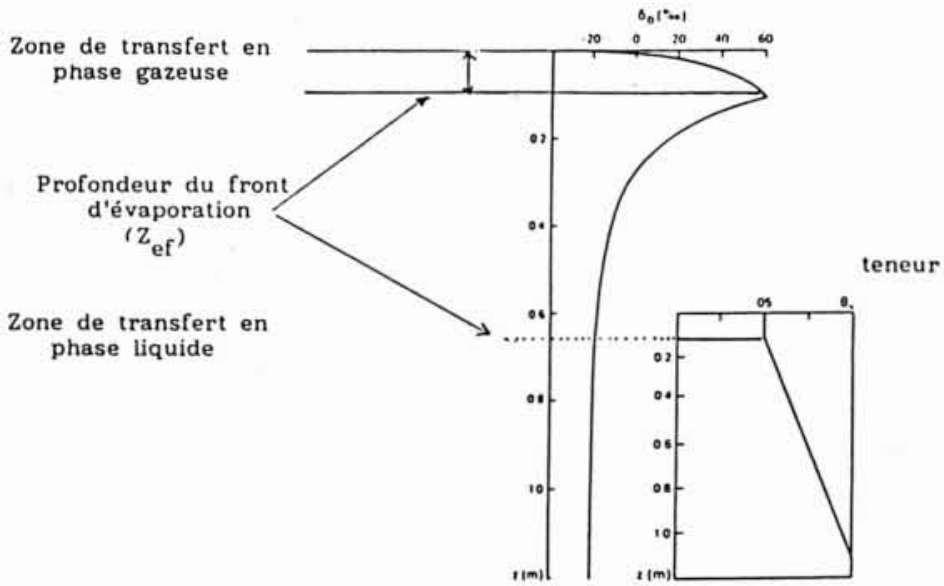

6. Profil isotopique théorique et profil de teneur en eau en fonction de la profondeur (d'après Barnes et Allison). 


\begin{tabular}{|c|l|c|c|c|c|}
\hline \multicolumn{2}{|c|}{$\mathrm{x}$ en $\mathrm{km}$} & 20 & 50 & 100 & 150 \\
\hline$\frac{h(x, t)}{h_{0}}$ pour & & & & & \\
& $t=10000$ ans & 0,8 & 0,5 & 0,2 & 0,06 \\
\cline { 2 - 6 } & $t=100000$ ans & 0,9 & 0,85 & 0,70 & 0,55 \\
\hline
\end{tabular}

Ces calculs montrent bien l'extension localisée d'une variation de niveau de courte durée (10 000 ans); elle est de $80 \%$ de l'amplitude de cette variation à $20 \mathrm{~km}$ et de $6 \%$ à $150 \mathrm{~km}$. Par contre pour une perturbation de plus longue durée, l'incidence même à une distance plus importante est encore significative ( $55 \%$ à $150 \mathrm{~km})$.

Avec une telle hypothèse, l'état piézométrique apparamment stabilisé des nappes superficielles du bassin sédimentaire du Sénégal ne serait plus le résultat d'un régime permanent comme on a tendance à le croire mais plutôt celui d'un régime transitoire à évolution très lente imperceptible à une échelle humaine.

\subsection{Le modèle de simulation :}

Toujours pour mieux tester l'hypothèse des variations du niveau marin et du climat, nous avons utilisé un modèle de simulation hydrogéologique; les unités modélisées sont :

- la nappe profonde à eau douce du Maestrichtien contenue dans des sables et grès;

- l'ensemble des nappes superficielles qui sont contenues dans des terrains d'âge différents mais qui sont en continuité hydraulique sur l'ensemble du bassin;

- dans la partie Sud une nappe intermédiaire contenue dans des terrains sablo-argileux de l'Oligo-Miocène.

Ces différentes nappes sont séparées par des niveaux semi perméables (argiles, marnes et calcaires compacts) dont l'épaisseur varie en moyenne de 50 à $400 \mathrm{~m}$. Le système modélisé sera ainsi tricouche et comprend près de 15000 mailles de 20000 à $5000 \mathrm{~m}$ de côté.

Le calage du modèle sera fait de la façon suivante :

- calcul en régime permanent avec les conditions morphoclimatiques régnant à 18000 ans $\mathrm{BP}$; niveau marin bas et climat sec. En effet nous avons par là supposé qu'à la fin de la dernière longue période de régression marine le niveau des nappes avaient atteint un régime plus ou moins stabilisé. Cette supposition du reste cohérente nous a ainsi permis d'éviter des simulations qui aurait porté sur des périodes de plus de 100000 ans;

- calcul en régime transitoire de 18000 ans BP à l'actuel. Pendant cette période seront prises en compte les variations de niveau marin et du climat, c'est ainsi que la côte atlantique et la bordure du fleuve Sénégal seront simulées comme des limites à potentiel imposé variable dans le temps;

- comparaison des états piézométriques observés et calculés pour la période actuelle.

Les cartes piézométriques de référence ont été :

- pour les nappes superficielles celles des années 1952-1954;
- pour la nappe profonde du Maestrichtien, celle de 1971;

- pour la nappe de l'Oligo-Miocène celle de 1981.

L'idéal aurait été bien sûr d'avoir des cartes piézométriques datant toutes de la même période et d'avant 1950 pour ne pas prendre en compte l'incidence des pompages. Mais comme ces derniers sont relativement faibles comparés aux possibilités des nappes et que l'évolution des niveaux des nappes superficielles n'est perceptible que sur de longues périodes, ces disparités seront sans inconvénients majeurs sur notre calage.

Bien qu'il nous a fallu une quarantaine de passage pour obtenir un état piézométrique des nappes compatible avec celui observé, signalons que la forme en creux de la nappe du Ferlo est apparue dès les premiers passages sans que nous ayons besoin de rechercher des jeux particuliers du paramètre. L'essentiel du calage a consisté par la suite à mieux affiner cette allure piézométrique par un ajustement des paramètres du modèle les moins bien connus: débit d'alimentation par les pluies et coefficient du transfert verticaux.

Les résultats du modèle ont ainsi permis de:

- confirmer l'hypothèse selon laquelle les variations du niveau marin et du climat pendant la dernière partie du Quaternaire pouvaient valablement expliquer les anomalies piézométriques observées dans la nappe du Continental Terminal du Sénégal;

- quantifier le bilan des flux d'apport par écoulement (latéral à partir des zones à piézométrie plus haute et par drainance à partir de la nappe profonde du Maestrichtien) dans la zone déprimée du Ferlo. En comparant ces flux à des flux d'évaporation sur nappe profonde qui seront calculés par d'autres méthodes, nous verrons par la suite dans quelle mesure une telle reprise par évaporation pourrait expliquer les anomalies en question;

- d'évaluer enfin de manière globale les ressources exploitables de la nappe profonde du Maestrichtien, principale réserve d'eau souterraine du Sénégal.

\section{L'hypothèse de la reprise par évapora- tion}

S'il a été relativement facile d'expliquer une dépression piézométrique telle que celle du Ferlo au Sénégal par une conjonction des variations du niveau marin et du climat pendant le Quaternaire, il est évident qu'il n'en serait pas de même pour les autres cas de nappes déprimées situées dans les bassins continentaux : au Mali et dans le bassin du Lac Tchad par exemple. L'éloignement de la mer par rapport à ces bassins rend cette hypothèse difficilement applicable. C'est certainement à cause de cette difficulté que l'hypothèse de l'évaporation, qui du reste revêt une importance toute particulière dans toute cette partie de l'Afrique, a été retenue comme explication plausible des cas de nappes en creux.

En plus de cet argument, d'autres pourraient militer en faveur de cette cause, c'est le cas par exemple de celui relatif à l'effet de la végétation sur les phénomènes 
évaporatoires en climat sec. En effet il semblerait que certaines espèces arbustives, pour s'adapter aux périodes sèches souvent longues des climats secs ou arides, développent en système racinaire puissant susceptible de prélever de l'eau à des nappes relativement profondes.

Malheureusement, la profondeur souvent assez importante (40 à $80 \mathrm{~m}$ en-dessous du sol) et la difficulté de mesurer directement les éventuels flux d'évaporation dont il serait question, rendent presque impossible une vérification rigoureuse de cette explication.

C'est ainsi que les seules possibilités d'estimations des flux d'évaporation sur nappes profondes reposent sur des méthodes isotopiques (Barnes, Allison, Fontes) qui utilisent l'enrichissement en sel et en certains isotopes qui se produit pendant le changement de phase (phase liquide à vapeur) consécutif à l'évaporation de l'eau de la nappe. En effet selon ces auteurs, la reprise par évaporation se fait de la nappe vers le sol dans un premier temps en une phase liquide suivie d'une phase gazeuse (figure 6); le niveau de transition entre ces deux phases ou front d'évaporation est caractérisé par:

- une augmentation de la salure consécutive au dépôt des sels contenus dans la phase liquide de l'eau évaporée; - un enrichissement en isotopes $\left({ }^{18} \mathrm{O} /{ }^{2} \mathrm{H}\right)$.

Signalons au passage que cette approche est différente de celle de certains auteurs selon lesquels l'augmentation de la salure de l'eau dans la partie déprimée des nappes en creux était un indice prouvant qu'il y avait reprise par évaporation dans la nappe.

Le flux d'évaporation $E$ sera alors :

$$
E(\mathrm{~mm} / \mathrm{an})=\frac{(1-\mathrm{ha}) \cdot N^{\mathrm{sat}} \operatorname{Dr}^{*}(p-\theta) z}{P \cdot Z_{\mathrm{ef}}}
$$

avec :

ha $=$ humidité relative de l'atmosphère à la surface du sol

$N^{\text {sat }}=$ concentration de la vapeur d'eau à saturation dans l'air saturé à la température du sol, au point d'évaporation

$\mathrm{D}_{\mathrm{v}}^{*} \quad=$ diffusivité de la vapeur d'eau dans l'air

$p \quad=$ porosité totale du sol

$\theta \quad=$ valeur moyenne de la teneur en eau

$Z \quad=$ coefficient de tortuosité du sol

$P \quad=$ masse volumique du liquide

$Z_{\text {ef }} \quad=$ profondeur du front d'évaporation.

A partir de cette formule et d'une extrapolation de données expérimentales (d'après J.C. Fontes à Beni Abbes en Algérie), nous calculons ${ }^{(1)}$ les flux d'évaporations suivants :

\begin{tabular}{|l|l|l|l|l|l|}
\hline $\begin{array}{l}\text { Profondeur de la } \\
\text { nappe en m }\end{array}$ & $10^{(2)}$ & 20 & 40 & 60 & 80 \\
\hline $\begin{array}{l}\text { Evaporation en } \\
\text { mm/an }\end{array}$ & $1,53^{(2)}$ & 0,65 & 0,26 & 0,14 & 0,08 \\
\hline
\end{tabular}

Pour une nappe profonde comme celle du Ferlo $(\approx 80 \mathrm{~m})$, ces flux restent relativement faibles pour expliquer à eux seuls une aussi importante dépression.
En effet il aurait fallu plus de 25000 ans pour que la vidange par évaporation puisse créer cette dépression piézométrique or avec une telle hypothèse, cette vidange devrait commencer après la dernière période humide, soit après 10000 ans $\mathrm{BP}$.

Enfin pour mieux situer la part de l'évaporation dans l'explication de cette nappe en creux du Ferlo, nous avons aussi comparé les flux précédents avec ceux d'écoulement en direction de la zone déprimée. En effet si l'évaporation devait être la cause principale de cette allure déprimée de la nappe, les flux qu'elle occasionne devraient être supérieurs à ceux résultant de l'apport par écoulement. Cette comparaison a donné le résultat suivant :

- flux d'écoulement latéral : $0,1 \mathrm{~mm} / \mathrm{an}$

- flux de drainance à partir de la nappe profonde : 0,06 $\mathrm{mm} / \mathrm{an}$

- flux de drainage à partir de la vallée du Ferlo : 0,02 $\mathrm{mm} / \mathrm{an}$

soit un flux global d'apport par écoulement de 0,18 $\mathrm{mm} / \mathrm{an}$ qui reste quand même supérieur à l'estimation précédente de l'évaporation $(0,08 \mathrm{~mm} / \mathrm{an})$.

\section{Les autres causes possibles :}

En plus des deux hypothèses précédentes qui ont été le plus souvent évoquées, les phénomènes de nappes en creux peuvent aussi être dû à des prélèvements humains ou à des causes d'origine géologique (tectonique). Pour le cas particulier du Sénégal, les derniers travaux d'inventaire de ressources et besoins en eau ont montré que les puisages étaient très insignifiants (traduits en lame d'eau ils sont souvent inférieurs à $10^{-2} \mathrm{~mm} / \mathrm{an}$ ) face à l'ampleur de la dépression du Ferlo.

Pour ce qui est des causes d'origine tectonique, les derniers mouvements de ce genre datent du Miocène si bien qu'ils ne doivent plus avoir une incidence sur la piézométrie actuelle; en plus leurs zones d'extension concordent mal avec celle des anomalies piézométriques observées.

\section{Les nappes déprimées de l'intérieur de l'Afrique Occidentale :}

Ce sont celles du Continental Terminal du Trarza en Mauritanie, du Gondo, du Delta intérieur du Niger et de l'Azaouad au Mali et enfin du bassin du Lac Tchad.

La difficulté majeure qui pourrait apparaître dans l'interprétation de ces cas de nappes déprimées et que à l'exception de celle du Trarza, l'hypothèse des variations

(1) Dans ces calculs, nous supposons la position du front d'évaporation fixe, seule varie avec la profondeur de la nappe la répartition des pressions dans le milieu non saturé sous-jacent à la nappe. La pression correspondante de la profondeur du front d'évaporation est celle du point de flétrissement habituel de plantes soit 15 bars.

(2) Valeurs expérimentales d'après J.C. Fontes à Beni Abbes en Algérie. 
du niveau marin est à écarter, la mer n'étant pas présente dans ces régions depuis le Tertiaire. Néanmoins un examen plus détaillé montre qu'il existe au sein de ces différentes nappes des disparités qui permettent de les classer par exemple en :

Nappes déprimées à niveau inférieur à celui de la mer actuel : il s'agit de nappes des bassins littoraux qui ont pu subir l'influence des variations passées du niveau de la mer, ce sont les nappes du Ferlo au Sénégal et du Trarza en Mauritanie.

Nappes fortement déprimées: l'amplitude de la dépression est de l'ordre de $80 \mathrm{~m}$; c'est le cas des nappes du Ferlo, du Trarza et du Gondo. Pour les deux premiers cas, l'explication des variations du niveau marin et du climat s'applique parfaitement car il s'agit de nappes de bassins littoraux. Pour ce qui est de la nappe du Gondo, des causes récentes d'origine géologique pourraient expliquer cette dépression de plus de $80 \mathrm{~m}$.

En effet une dissolution des niveaux calcaires dolomitiques a provoqué dernièrement des effondrements qui ont donné naissance au cratère d'Irma profond de $55 \mathrm{~m}$. En outre une synthèse de mesures de niveau (L. Bourguet, 1974), bien que fragmentaire, semble montrer que les niveaux de la nappe du Gondo seraient en train de monter. Cela confirmerait l'hypothèse des causes tectoniques; en effet dans un tel schéma la nappe du Gondo serait en train de se remplir par un écoulement centripète très lent au lieu de se vidanger sous l'effet de l'évaporation.

Nappes de dépression moindre: c'est le cas des nappes de l'Azaouad, du delta du Niger et du bassin du Lac Tchad. Dans tous ces cas la dépression est de l'ordre de $40 \mathrm{~m}$ et à cette profondeur, il n'est pas exclu que l'évaporation puisse avoir une incidence significative dans l'explication de ces anomalies piézométriques. A cette profondeur, l'évaporation estimée à partir des calculs précédents est de $0,26 \mathrm{~mm} / \mathrm{an}$. Par ailleurs, l'influence des variations des conditions hydroclimatiques anciennes n'est pas à exclure. Ces nappes contenues dans des formations peu perméables et peu diffuses sont essentiellement alimentées à partir du ruissellement d'une surface qui aurait bien pu subir les variations climatiques du Quaternaire. En plus, les cours d'eau ou lac qui servent de source d'alimentation à ces nappes et qui parfois se raccordent à la mer ont aussi certainement subi des variations de niveaux. C'est ainsi qu'il a été noté des variations de niveaux lacustres dans le $\mathrm{Bahr} \mathrm{El}$ Ghazal (bassin du Lac Tchad) de $60 \mathrm{~m}$ entre 6000 ans BP et l'actuel (M. Servant, 1983).

\section{Alimentation des nappes superficielles - Réserve utile du sol}

La mise en œuvre du modèle a été une occasion nous ayant permis de nous rendre compte de la difficulté liée à l'estimation de la part de la pluie qui s'infiltre vers les nappes.

Les débits d'alimentation susceptibles de reproduire une piézométrie des nappes compatible avec celle observée ont été de loin inférieurs à ceux qu'on aurait pu calculer avec des modèles de bilan hydrologiques habituels. Cette disparité est certainement le résultat d'une utilisation non appropriée de la notion de réserve utile du sol. En effet celle-ci représente le stock d'eau emmagasiné dans les premières tranches de sol où sont censés se produirent les échanges entre le sol et l'atmosphère, soit donc généralement une épaisseur de l'ordre du mètre. C'est pour cette raison que souvent dans les calculs de bilan hydrologique, les valeurs de réserve utile adoptées dépassent rarement une lame de 100 à $150 \mathrm{~mm}$. Pourtant même si de telles valeurs ou d'autres encore plus faibles donnent des résultats satisfaisants en climat tempéré, il s'avère tout à fait justifié qu'elles soient corrigées en hausse pour les climats arides ou secs où la zone d'échange entre nappes et atmosphère y est nettement plus épaisse.

C'est ainsi que :

- J.C. Fontes a montré en Algérie que l'évaporation est perceptible sur des nappes profondes de $10 \mathrm{~m}$;

- C. Charreau à travers une étude sur la dynamique de l'eau dans des sols du Sénégal a aussi montré que la dessication du sol en saison sèche se manifestait jusqu'à au moins $4 \mathrm{~m}$ de profondeur. Il a par ailleurs quantifié la variation de la capacité de rétention du sol en fonction de la profondeur; celle-ci correspond à quelque chose près à la variation de la réserve utile. C'est ainsi donc que la rétention passe de $59 \mathrm{~mm}$ à $40 \mathrm{~cm}$ de profondeur à $161 \mathrm{~mm}$ à $1 \mathrm{~m}$ et $314 \mathrm{~mm}$ à $2 \mathrm{~m}$ pour des sols plus ou moins sablo-argileux.

Ces réflexions et résultats nous ont alors permis d'ajuster les valeurs de réserves utiles et d'obtenir avec un modèle de bilan plus adapté des débits d'alimentation certes plus faibles mais aussi plus cohérents comparés à la piézométrie restituée.

Nous nous sommes aussi rendu compte que l'alimentation des nappes est le plus souvent liée à l'existence d'un réseau de ruissellement et que même avec une pluviométrie de $800 \mathrm{~mm}$ il est possible qu'il n'y ait pas une infiltration directe et généralisée des eaux de pluies.

\section{Conclusion}

Par le biais de ce travail, nous nous sommes rendus compte que les différents cas de nappes en creux de l'Afrique Occidentale ne pouvaient pas être tous expliqués par la même et unique cause. Pour cette raison, nous pensons qu'une explication générale de ce phénomène réside dans une approche pluridisciplinaire qui consistera à chercher pour chaque cas la cause la plus significative parmi celles possibles. 
Il nous est aussi apparu que ces nappes, compte tenu de leur diffusivité très faible, ne pouvaient pas être en régime permanent sous les conditions hydrologiques actuelles. En conséquence cela impose pour ce qui est de l'étude de leur comportement hydrodynamique et de leurs conditions d'exploitation une vision plus large dans le temps.

\section{Référence}

DiEng B., 1987. - Paléohydrogéologie et hydrogéologie quantitatives du bassin sédimentaire du Sénégal. Essai d'explica. tion des anomalies piézométriques observées. Thèse de doctorat de l'Ecole des Mines de Paris. Ce document contient plus de 100 références relatives au sujet ci-dessus abordé.

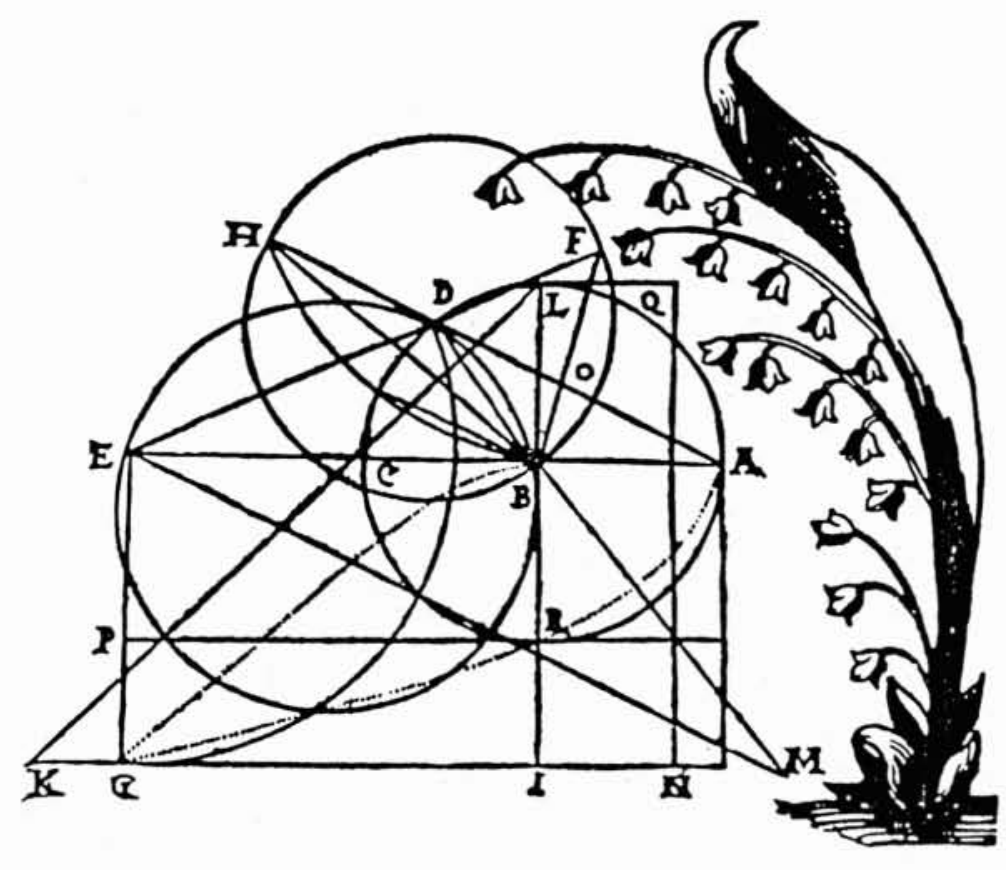

Adresse de l'auteur

Monsieur Babacar Dieng

Ecole inter-états d'ingénieurs de l'équipement rural BP 7023

Ouagadougou (Burkina Fasso)

Tél. 19-226-33-35-28 (ou 29)

ou

Direction des études hydrauliques

Ministère de l'hydraulique

BP 4021

Dakar (Sénégal)

Tél. 19-221-22-21-54 